\title{
Vertical Jump to Female \& Male Basketball Players in Albania
}

\author{
Orest Shytaj \\ Marin Barleti University
}

\section{Abstract}

Vertical jump is one of the basic elements in collective sports such as volleyball, basketball, football, etc. The reason of this study is the evaluation of this element and other parameters of the Albanian National Championship basketball players female \& male. From the specificity of their training, there are differences in the physical qualities of individual performance, where one of the elements is vertical jumping. The performance profiling of basketball players is important to distinguish the anthropometric and physical characteristics from other sports. Basketball players were tested in different periods before and after the training with their coaches. Subjects were 59 Female (F) mean age 18.1 years old and 60 Male (M) mean age 18.8 years old. They evaluated us; Body Height (BH), Body Mass (BMI \%), Body Weight (BW), Squat Jump (SJ), Countermovement Jump (CMJ) and Drop Jump 40cm (DJ). From the obtained data of the test was observed even the effect of the training that these athletes have done for the jumping level, assessment of explosive strength and maximum power. Differences were seen to individual basketball players between two periods, before and after the testing. The evaluation of these tests is one of the key features of a vertical jump as one of the specific technical element in the game on air in the sport of basketball. The obtained results suggest changes in sporting performance of "vertical jump" in the force settings, speed and power.

Keywords: basketball, squat-countermovement jump, drop jump, female \& male

\section{Introduction}

Basketball is one of the most popular sports in the world but also quite developed in our country Albania. Basketball players during their game, in addition to various runs, must also develop vertical jumping in catching balls on the table or even fighting in the air with the opponent. Plyometric training [1] is widely used to improve jumping ability, especially in sports such as volleyball and basketball $[2,3,4,5]$. Volleyball and Basketball sports have different performances for their own game characteristic, so the training is specific where there are differences in the physical qualities of individual and team performance. Methods plyometric and resistance training are usually referred to in the literature as improving the most powerful strength 
characteristics (explosive strength) in basketball players. In basketball, the ability to generate maximal strength levels in the shortest period of time (muscular power) is necessary to gain high sport performance levels [6]. Moreover, agility is a vital component for the success in basketball players [7].So the meaning of the vertical jump element is basically the measurement of the strength, strength, speed of the lower extremities. Numerous scholars have found that high jumps can be improved through applications of plyometric exercises. The best possible perfection of a vertical jump is achieved with training with the purpose of increasing the height of the jump, the high degree of muscular activity that is achieved by increasing the loads in the phase of extension of the vertical jump. Therefore, the purpose of this study is to identify the performance of vertical dance and the comparison between the sexes of some young basketball players in Albania by using the tests squat jump (SJ), countermovement jump (CMJ) and drop jump (DJ). Individual differences in physical fitness parameters among players have been attributed to long-term sport training specificity [8]. To assess the vertical jump is needed the application of Bosco tests such as Drop Jump [9], that made possible the definition of the height of the optimum fall from which the player takes \wins the maximum jumping and the connection between the height of the fall, the time of contact and flight-time. To developed this study we have used the facilities of the University of Sports in Tirana and its respective laboratories of Physiology and Biomechanics where tests are developed with advanced equipment and GFRP "Leonardo" platform.

\section{Methodology}

In understanding with coaches of the basketball teams we based our study on the female \&male in the period before and after a preparatory mini phase in the period before and after the second phase of the National Championship. During the study players have been under our observation for the performance of the training program with their coaches. Training sessions have been regular, 5 times a week with duration of 1 hour and 30 min each time. In this study participate these basketball players and they were tested in these parameters shown in table 1 ;

Table1 Anthropometric Measurements (Basketball players)

\begin{tabular}{|l|l|l|}
\hline Nr; & $\begin{array}{l}\text { FEMALE } \\
\text { (F) 59 }\end{array}$ & $\begin{array}{l}\text { MALE } \\
\text { (M) 60 }\end{array}$ \\
\hline Mean Age & 18.1 & 18.8 \\
\hline Body Height (BH) cm & $182.4 \mathrm{~cm}$ & $195.9 \mathrm{~cm}$ \\
\hline Body Wight (BW) kg & $71.8 \mathrm{~kg}$ & $83.2 \mathrm{~kg}$ \\
\hline Body Mass (BMI)\%kg/m ${ }^{2}$ & $21.55 \%$ & $20.37 \%$ \\
\hline
\end{tabular}




\subsection{Protocols of the Test Performed}

- SJ (squat jump) - The vertical jump from the initial position starts when the athlete has his/her legs refracted to $90^{\circ}$ and hands positioned on the waist. There have been realized 3 jumps and has been taken the best.

- CMJ (Countermovement Jump) is similar to SJ, but the athlete begins the testing by

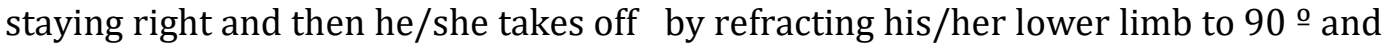
hands placed on the waist (3 jumps). Tests were developed on Muscle Lab Jump.

- DJ (drupe jump) from $40 \mathrm{~cm}$ cube in Leonardo® Ground Force Reaction Plate (GRFP-Novotec Medical, Pforzheim, Germany).

- Calculation (CMJ-SJ) x 100/CMJ). The capacity of reusing the accumulated energy as a result of the elastic stretch that precedes the muscular contraction.

During squat jumps with a knee flexion to $90^{\circ}$ players were instructed to perform a maximal vertical jump and were not allowed to use any motion, forward flexion before jumping. All volleyball players were tested in the same conditions, with a preliminary general stretching of $10 \mathrm{~min}$. The basketball players then developed an individual warm-up of 10 min before starting the specific vertical jump performance test in the Squat Jump-SJ, Countermovement Jump-CMJ and Drop Jump-DJ at the throw height from $40 \mathrm{~cm}$ cubes [10]. Basketball players are initially recommended for each performance of the jump test how it should proceeded.

\section{Results}

Table 2 shows the average team data of female and male basketball players taken under observation. The values obtained clearly show the level of the players in Albania.

Table2. Data obtained from Ergo Jump Muscle Lab Jump (female \& male)

\begin{tabular}{|l|l|l|l|l|}
\hline Basketball players & \multicolumn{3}{|l|}{ FEMALE } & MALE \\
\hline & Test 1 & Test 2 & Test 1 & Test 2 \\
\hline Squat Jump & 27.7 & 29.7 & 33.4 & 39.8 \\
\hline Countermovement Jump & 33.9 & 35.2 & 39.2 & 45.9 \\
\hline (CMJ-SJ)x100/CMJ\% & 18.07 & $15.50 \%$ & $18.10 \%$ & $14 \%$ \\
\hline
\end{tabular}

Table.3. Data of the tests done with GFRP “Leonardo” (female \& male)

\begin{tabular}{|l|l|l|l|l|l|l|l|l|}
\hline & \multicolumn{3}{|l|}{ Squat Jump Test } & \multicolumn{3}{l|}{ Countermovement Jump Test } \\
\hline & Test & Test & Test & Test & Test & Test & Test & Test \\
& 1 & 2 & 1 & 2 & 1 & 2 & 1 & 2 \\
& Fmax & Fmax & Pmax & Pmax & Fmax & Fmax & Pmax & Pmax \\
\hline
\end{tabular}




\begin{tabular}{|l|l|l|l|l|l|l|l|l|}
\hline FEMALE & 1.38 & 1.48 & 2.73 & 2.97 & 1.46 & 1.53 & 2.81 & 2.96 \\
\hline MALE & 1.55 & 1.6 & 3.52 & 3.57 & 1.51 & 1.75 & 3.32 & 3.71 \\
\hline
\end{tabular}

\section{Discussion - Analysis}

From the data shown in tab.2,3 it seems clear in comparison and with different references from the foreign literature, female and male basketball players have low jumping levels ;referred by [11] (SJ $37 \mathrm{~cm}, \mathrm{CMJ} 41 \mathrm{~cm}$ for girls) ) (SJ boys $47 \mathrm{~cm}, \mathrm{CMJ}$ 53). But in both teams there have been male and female individuals who have improved during the training with the coaches. It is clearly seen in younger athletes. For a long time we thought that CMJ jump would provide the muscle elasticity measure, while today it is found that this test provides the rapid force measure of the jump. The differences between the two tests CMJ-SJ of the athletes in teams is the "elasticity index". Good capacity used by the elastic energy corresponds to $8-10 \mathrm{~cm}$. Difference between these two tests is called "The index of fast power" [11].The difference in \% of (CMJ-SJ) the calculation of the elasticity index. Elastic energy (CMJSJ) $x 100 / C M J$ the capacity for subsequent reuse of accumulated elastic energy resulting from the elastic stretch preceding muscular contraction. From the data obtained before and after the training of the basketball players there is still exploited elastic energy. We think that there is more to be clarified and developed in the training plan that the coaches use to increase the vertical jump to basketball players. Vertical jump assessment has been widely used as an alternative to the maximum straightforward assessment of strength and power of the lower extremities [12]. This platform consists in obtaining more data to the evaluation of the basketball players, force (F max $\mathrm{kN}$ ) and power (P max $\mathrm{kW}$ ), during the vertical jump. This platform clearly shows and identifies all the testing protocol of the vertical jump. Technical good used efficiency (EFI \%) during the vertical jump highlights the general preparation that this sports has in lower extremities (legs) and the usage of the elastic energy of the muscle complex. From the observation and the conversation with the coaches of these two teams we realized that despite the general athletic-technicaltactical preparation they used different plyometric exercises to increase the vertical jump.

Table.4" Data of the tests done with GFRP "Leonardo" (female \& male) DJ $40 \mathrm{~cm}$

\begin{tabular}{|c|c|c|c|c|c|c|c|c|c|c|}
\hline \multirow[t]{2}{*}{$\begin{array}{l}\text { Basketb } \\
\text { all } \\
\text { Players }\end{array}$} & \multicolumn{2}{|c|}{$\mathrm{F} \max \mathrm{kN}$} & \multicolumn{2}{|c|}{$\mathrm{P} \max w / \mathrm{kg}$} & \multicolumn{2}{|c|}{$\begin{array}{l}\text { Time } \\
\text { Contact(TC) } \\
\text { s }\end{array}$} & \multicolumn{2}{|c|}{$\begin{array}{l}\text { Air } \\
\text { Time(TA)s }\end{array}$} & \multicolumn{2}{|c|}{ TA/TC s } \\
\hline & \multicolumn{2}{|c|}{$\begin{array}{l}\text { Test 1- } \\
\text { Test 2 }\end{array}$} & \multicolumn{2}{|c|}{$\begin{array}{l}\text { Test 1-Test } \\
2\end{array}$} & \multicolumn{2}{|c|}{$\begin{array}{l}\text { Test 1- } \\
\text { Testi2 }\end{array}$} & \multicolumn{2}{|c|}{$\begin{array}{l}\text { Test 1-Test } \\
2\end{array}$} & \multicolumn{2}{|c|}{$\begin{array}{l}\text { Test 1- } \\
\text { Test2 }\end{array}$} \\
\hline $\begin{array}{l}\text { FEMAL } \\
\text { E }\end{array}$ & $\begin{array}{l}3.3 \\
1\end{array}$ & $\begin{array}{l}3.1 \\
8\end{array}$ & 30.8 & 33.4 & $\begin{array}{l}0.30 \\
4\end{array}$ & $\begin{array}{l}0.30 \\
3\end{array}$ & 0.48 & $\begin{array}{l}0.93 \\
4\end{array}$ & $\begin{array}{l}1.6 \\
9\end{array}$ & $\begin{array}{l}1.7 \\
4\end{array}$ \\
\hline
\end{tabular}




\begin{tabular}{|l|l|l|l|l|l|l|l|l|l|l|}
\hline MALE & 3.3 & 3.1 & 14.4 & 33.6 & 0.41 & 0.32 & 0.49 & 0.51 & 1.3 & 1.5 \\
& 1 & 2 & 5 & 3 & 9 & 8 & 9 & 2 & 4 & 9 \\
\hline
\end{tabular}

Graffic.1.Drop Jump $40 \mathrm{~cm}$ (female \& male)

TA/TCs Test-1

TA/TCs Test-2

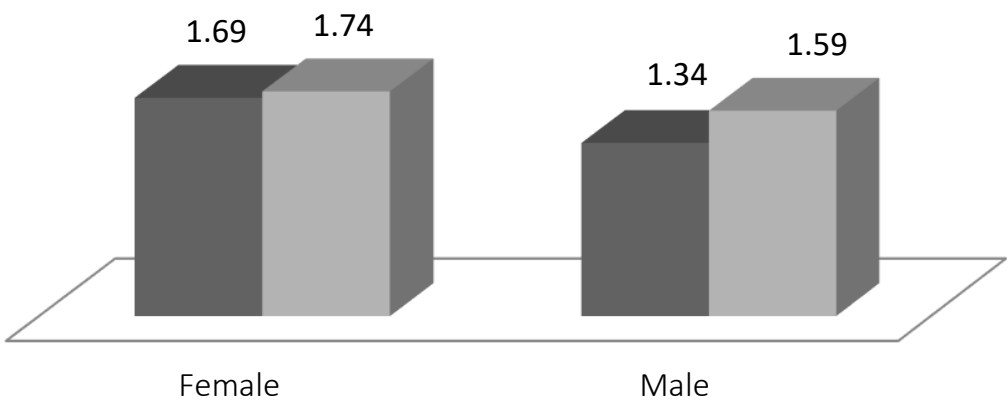

This is a jump that is standardized to fall from a height, contact with the falling ground and knees at an angle of $90^{\circ}$, without interruption, in a vertical jump to the maximum with a minimum foot contact with the GRFP force platform (Specific plyometric indicator). The Drop Jump test offers a progressive increase of the height of the falling cube from $20 \mathrm{~cm}, 40 \mathrm{~cm}, 60 \mathrm{~cm}$ and $80 \mathrm{~cm}$ near a force platform GRFP. This test assesses the explosive strength of the lower limbs. The test represents the "ExplosiveReactive-Ballistic Force" and in particular, the neuromuscular capacity to develop very high values of force during the stretch-contraction cycle [9]. In this test we used fall from $40 \mathrm{~cm}$ high cube in both volleyball teams. The test provides as an objective the progressive increase in the decline faze, contact time (TCs), the residence time on air (TAs) and has also the objective to show the highest determining result of the vertical jump. Bosco has come to the conclusion that to have significantly results TA/TC on DJ test. The results of the study as outlined above for women and men show that using the DJ test we can improve the vertical jump. By comparing the first and second DJ test, the results show the higher increase of the sportive performance in maximal force and maximal power in male volleyball players than female, especially in particular individuals. As a result of improved power and force is also increased the vertical jump shown in the declining contact DJ faze with the time of flight, expressed in seconds. DJ exercises are plyometric exercises commonly used to increase explosive power through stretching-shortening cycle muscle activity. Thus it helps to gain the greatest power output and energy output in the concentric phase [11]. Optimal determination of DJ jump height is important in training volleyball players. Despite the large number of plyometric studies, few studies on exercise have directly compared the effectiveness of plyometric, DJ training and CMJ training. Various authors have found that high jumps can be improved through plyometric 
exercises. Statistical analysis was performed using IBM Statics 20. As a working environment between groups: ANOVA with repeated measurements with time factor and/or group. The results of descriptive statistical analysis for the dependent variable "TA / TC" presented in table 4 showed that there is progress in increasing performance in this test of both groups of volleyball players. Some authors have reported [13] that commanded plyometric training shows that plyometric exercises are effective in increasing the volleyball, speed and dexterity of volleyball players. In Test 2 measurements there is an average significant increase in seconds for both females and males.

\section{Conclusion}

We think that there is more to be clarified and developed in the training plan that coaches use to improve the vertical jump to basketball players. . DJ test evidence that is a more effective way to increase the output power and the jumping performance. From the obtained results of the study we have arrived on these conclusions: Vertical jump SJ and CMJ done in Ergo jump and with the implementation of the method Bosco test 1 and test 2 training shows that tested basketball players have yet untapped and well-used energy. We think that in terms of training from coach's plyometric exercises to increase the explosive power in vertical jump have not been done regularly with a detailed program. But for female and even for male players the training has been efficient. Max force (F max) and max power (P max) are relatively poor values (low) compared with the results that a basketball player should have during a vertical jump. Practicing these tests, we think that in the sport of basketball they are a necessity of a coaching advancement for the ages to have a better performance of their basketball jumping for both men and women.

\section{References}

[1] Wilt, F. Plyometrics-What it is-How it works. Athl. J.55(76):89-90. 1975.

[2] Blattner, S., and L. Noble. Relative effects of isokinetic and plyometric training on vertical jumping performance. Res Q. 50:583-588. 1979.

[3] Brown, M.E., J.L. Mayhew, and M.a. Boleach. Effect of plyometric training on vertical jump performance in high school basketball players. J. Sports Med. Phys. Fitness 26:1-4. 1986.

[4] Fatouros, G.I., Z.a. Jamurtas, D. Leontsini, K. Taxildaris, N. Aggelousis, N. Kostopoulos, and P. Buckenmeyer. Evaluation of plyometric exercise training, weight training, and their combination on vertical jumping performance and leg strength. J. Strength Cond. Res. 14:470-476. 2000.

[5] Hewett, T.E., a.L. Stroupe, T.a. Nance, and F.R. Noyes. Plyometric training in female athletes. Decreased impact forcforces and increased hamstring torques. Am. J. Sports Med. 24: 765-773. 1996.

[6] In basketball, the ability to generate maximal strength levels in the shortest period of time (muscular power) is necessary to gain high sport performance levels . 
[7] Arazi H, Coetzee B, Asadi A (2012) Comparative effect of land and aquatic based plyometric training on the jumping ability and agility of young basketball players. S Afr J Res Sport Phys Edu Rec 34:1-14

[8] Izquierdo,M.,K.Hakkinen,J.J.Gonzalez-

Badillo,J.Ibanez\&E.M.Gorostiaga,2002.Effects of long-term training specificity on maximal strength and power of the upper and lower extremities in athletes from different sports. Eur. J. Appl. Physiol. 87:264-271.

[9] Cometti G., \& Cometti D., 2009). La Pliometria (origini, teorie, allenamento) (2nd ed.) Tivoli: Italy.60-67.

[10] Carmelo Bosco,2006. Ph.D."La forza muscolare"-Aspetti Fisiologici ed Applicazioni Pratiche" Capitolo IV, La Forza Esplosiva ,pg103-110.

[11] Komi ,P.V,1992.Stretch-shortening cycle. In: P.V. Komi (ed.) Strength and Power in Sport. Blackwell Scientific Publications, Oxford,;pp.169-179

[12] Hara, M., Shibayama, A., Takeshita, D., \& Fukashiro, S.,2006.The effect of arm swing on lower extremities in vertical jumping. J Biomech 39: 2503-2511.

[13] Voelzke,Mathias\&Stutzig,Norman and Thorhauer, Hans-Alexander and Granacher, Urs. 2012.Promoting lower extremity strength in elite volleyball players: effects of two combined training methods. J Sci Med Sport,Volum15,Nr 5;page 457-462. 